\title{
Risk Factors for Symptomatic Gallstone Disease and Gallstone Formation After Bariatric Surgery
}

\author{
Sylke Haal ${ }^{1,2}$ (1) - Maimoena S. S. Guman ${ }^{1,3} \cdot$ Sjoerd Bruin $^{4} \cdot$ Ruben Schouten $^{5} \cdot$ Ruben N. van Veen $^{6} \cdot$ Paul Fockens $^{2}$. \\ Marcel G. W. Dijkgraaf ${ }^{7}$ - Barbara A. Hutten ${ }^{8} \cdot$ Victor E. A. Gerdes $^{1,3} \cdot$ Rogier P. Voermans $^{2}$
}

Received: 3 December 2021 / Revised: 2 February 2022 / Accepted: 3 February 2022 / Published online: 10 February 2022

(c) The Author(s) 2022

\begin{abstract}
Purpose Patients who undergo bariatric surgery are at risk for developing cholesterol gallstones. We aimed to identify risk factors that are associated with symptomatic gallstone disease and gallstone formation after bariatric surgery.

Materials and Methods We included participants of the UPGRADE trial, a multicenter randomized placebo-controlled trial on the prevention of symptomatic gallstone disease with ursodeoxycholic acid (UDCA) after bariatric surgery. The association between patient characteristics and symptomatic gallstone disease, and gallstone formation was evaluated using logistic regression analysis.

Results Of 959 patients, 78 (8\%) developed symptomatic gallstone disease within 24 months. Risk factors were the presence of a pain syndrome (OR 2.07; 95\% CI 1.03 to 4.17) and asymptomatic gallstones before surgery (OR 3.15; 95\% CI 1.87 to 5.33 ). Advanced age (OR $0.95 ; 95 \%$ CI 0.93 to 0.97 ) was protective, and UDCA prophylaxis did not reach statistical significance (OR $0.64 ; 95 \%$ CI 0.39 to 1.03 ). No risk factors were identified for gallstone formation, whereas advanced age (OR 0.98; $95 \%$ CI 0.96 to 1.00 ), statin use (OR $0.42 ; 95 \%$ CI 0.20 to 0.90 ), and UDCA prophylaxis (OR 0.47 ; $95 \%$ CI 0.30 to 0.73 ) all reduced the risk.

Conclusion Young patients with a preoperative pain syndrome and/or asymptomatic gallstones before bariatric surgery are at increased risk for symptomatic gallstone disease after surgery. Whether statins, either alone or in combination with UDCA prophylaxis, can further reduce the burden of gallstones after bariatric surgery should be investigated prospectively.
\end{abstract}

Keywords Gallstone formation $\cdot$ Symptomatic gallstone disease $\cdot$ Risk factors

\section{Introduction}

Rapid weight loss after bariatric surgery is a major risk factor for cholesterol gallstone disease [1]. Among patients who undergo bariatric surgery, up to $40 \%$ develop gallstones

\section{Key Points}

- Protective factor for gallstone disease after bariatric surgery: advanced age

- Risk factors for gallstone disease: presence of pain syndrome \& asymptomatic gallstones

- Protective factors for gallstone formation: UDCA, advanced age

$\&$ statin use

- No risk factors were identified for gallstone formation after bariatric surgery

Sylke Haal

s.haal@amsterdamumc.nl

Extended author information available on the last page of the article
[2-4], and approximately $8 \%$ to $15 \%$ become symptomatic requiring a cholecystectomy [5-7]. Despite extensive research, it is currently not possible to predict the risk for gallstones in the individual bariatric patient. Most previous studies did not identify any preoperative risk factors [2, 3, 8-22], whereas others found inconsistent associations between gender, age, ethnicity, the presence of comorbidities (such as pain syndrome, hypertension, type 2 diabetes, dyslipidemia, fatty liver disease and insulin resistance), the use of statins, or type of surgery on the one hand, and symptomatic gallstone disease or gallstone formation after bariatric surgery on the other hand [4, 23-39]. Although the postoperative weight loss predisposes bariatric patients to develop gallstones, it cannot be predicted beforehand. Furthermore, it remains uncertain whether the variation in speed and amount of the weight loss are responsible for why some patients form gallstones extremely rapid after bariatric surgery (within in 6 weeks), while others do not. 
The purpose of this study was to investigate associations between patient characteristics and symptomatic gallstone disease, and gallstone formation after bariatric surgery using data from the UPGRADE trial, a large randomized placebocontrolled trial [40, 41].

\section{Methods}

\section{Study Design and Patients}

The source population for this prospective cohort study comprised the participants of the UPGRADE trial (Netherlands Trial Register, NL5954). The protocol and results of this trial have been described elsewhere [40, 41]. In summary, the UPGRADE trial was a multicenter, randomized, placebo-controlled, double-blind superiority trial conducted in the Netherlands between January 2017 and November 2020. Patients with morbid obesity, an intact gallbladder, and scheduled for laparoscopic Roux-en-Y gastric bypass (RYGB) or sleeve gastrectomy were included. Key exclusion criteria were the presence of symptomatic gallstone disease before bariatric surgery, prior bariatric surgery, and prior gallbladder surgery. Additional exclusion criteria are listed in the trial protocol [40]. All patients received a preoperative ultrasound before randomization to determine the presence of asymptomatic gallstones. The result of the ultrasound was blinded for the patients and treating physicians. Patients were randomly assigned to receive either $900 \mathrm{mg}$ ursodeoxycholic acid (UDCA) daily for 6 months or placebo, and were followed for 24 months. The gallbladder ultrasound was repeated at 24 months (window 18 to 30 months). All patients provided written informed consent. The ethical committee of the Slotervaart Hospital and Reade (Amsterdam, the Netherlands) and the boards of directors at each hospital approved the trial protocol before local conduct started.

\section{Data Collection}

Data were collected by the central study coordinators ( $\mathrm{SH}$ and MG) in electronic case report forms using Castor EDC [42]. Preoperatively, data on demographics, presence of comorbidity, and preoperative medication use were collected. Hypertension, type 2 diabetes, and pain syndrome were defined as earlier described [30]. Dyslipidemia was defined as the presence of at least one of the following: known dyslipidemia, use of lipid-lowering drugs, a high-density lipoprotein cholesterol $\leq 0.9 \mathrm{mmol} / \mathrm{L}$, lowdensity lipoprotein cholesterol $\geq 5.0 \mathrm{mmol} / \mathrm{L}$, total cholesterol $\geq 6.5 \mathrm{mmol} / \mathrm{L}$, or triglycerides $\geq 5.0 \mathrm{mmol} / \mathrm{L}$. The individual potency of each statin was determined according to the meta-analysis by Law et al. [30, 43]. Ethnicity was self-reported by the trial participants. Categories consisted of Dutch, the four major non-Western ethnic groups in the Netherlands (Surinamese, Dutch Caribbean, Moroccan, Turkish), a category for all other ethnic groups, and a category for individuals with multiethnic backgrounds. Postoperatively, information on weight loss was gathered at 6,12 , and 24 months. Percent total weight loss (\%TWL) was calculated by the following formula: $\% \mathrm{TWL}=[($ initial weight - postoperative weight)]/initial weight] $\times 100$.

\section{Study Outcomes}

The primary endpoint was the occurrence of symptomatic gallstone disease, defined as hospital admission or hospital visit for symptomatic gallstone disease within 24 months after bariatric surgery. Symptomatic gallstone disease comprised acute biliary pancreatitis, acute cholecystitis, choledocholithiasis, cholangitis, and biliary colics. Each different etiology of symptomatic gallstone disease was diagnosed based on a specific combination of predefined clinical characteristics, laboratory results, and imaging findings. The definitions are described in detail in the published trial protocol [40] Primary endpoint adjudication was done by a blinded adjudication committee. In patients without gallstones before surgery, we assessed the formation of gallstones and/or sludge by ultrasound (window 18 to 30 months). The group of patients with gallstones and/or sludge on the follow-up ultrasound consisted of symptomatic and asymptomatic patients.

\section{Statistical Analysis}

Logistic regression analysis was used to evaluate associations between patient characteristics and (1) the occurrence of symptomatic gallstone disease and (2) the formation of gallstones and/or sludge after bariatric surgery. Variables with known clinical importance and/or with a $p$-value of 0.10 or less in the univariable analysis were selected for multivariable analysis. Stepwise backward elimination was used to derive a final model: In each subsequent step, the least significant variable in the model was removed until all remaining variables had individual $p$-values smaller than 0.10 . To take into account the effect of the intervention of the UPGRADE trial, three different strategies were conducted. First, the intervention was included in both multivariable models. Second, both analyses were repeated in patients assigned to placebo. Third, interactions between the intervention and patient characteristics were explored. In case of a significant interaction, the interaction term was included in the multivariable model. All statistical tests were two-sided and a p-value of less than 0.05 was considered significant. Statistical analyses were performed using SPSS statistics for Windows (version 26, Armonk, NY: IBM Corp). 


\section{Results}

\section{Study Population}

The study population of the UPGRADE trial consisted of 967 patients, of whom 959 were available for primary endpoint assessment at 24 months (Fig. 1). Demographic and clinical characteristics are shown in Table 1 . The mean age ( \pm standard deviation) was $45.1 \pm 11.1$ years, and most patients were female (766 [80\%]) and had a Dutch background (734 [77\%]). The mean weight and body mass index at surgery were $116.0 \pm 18.0 \mathrm{~kg}$ and $40.2 \pm 4.7 \mathrm{~kg} / \mathrm{m}^{2}$, respectively. Hypertension was the most common comorbidity (468 [49\%]), and about one-fifth (186 [19\%]) of the patients was diagnosed with asymptomatic gallstones before surgery. The majority received a laparoscopic RYGB (881 [92\%]), and half of the patients was assigned to receive UDCA. Mean \%TWL was $23.0 \pm 5.4$ at 6 months, $29.4 \pm 7.4$ at 12 months, and $29.4 \pm 8.9$ at 24 months. A subset of the study population-consisting of 669 patients without gallstones before surgery who received a postoperative ultrasound—shared all other characteristics (Table 1).

\section{Factors Associated with Symptomatic Gallstone Disease}

During 24 months of follow-up, 78 patients (8\%) developed symptomatic gallstone disease after a median [IQR] of 316 [184 to 477] days. In patients with asymptomatic gallstones before surgery, 27 (15\%) of 186 patients developed symptomatic gallstone disease. The results of the univariable and multivariable logistic regression analyses for the outcome occurrence of symptomatic gallstone disease are shown in Table 2. The presence of asymptomatic gallstones before surgery (odds ratio [OR] 2.40; 95\% confidence interval $[\mathrm{CI}], 1.46$ to $3.95 ; p<0.001)$ and a higher $\% \mathrm{TWL}$ at 24 months (OR 1.03; 95\% CI 1.00 to $1.06 ; p=0.04$ ) were significantly associated with an increased risk for symptomatic gallstone disease after bariatric surgery in the univariable analysis. Advanced age (OR 0.96; 95\% CI, 0.94 to $0.98 ; p<0.001$ ) and the use of statins (OR $0.38 ; 95 \%$ CI, 0.16 to $0.89 ; p=0.03$ ) were both significantly associated with a reduced risk. No dose-effect relationship was observed between the intensity of the statins and the occurrence of symptomatic gallstone disease $(p=0.32)$. In the multivariable analysis, the presence of a pain syndrome (OR 2.07; 95\% CI 1.03 to $4.17 ; p=0.04$ ) and the presence of asymptomatic gallstones before surgery (OR $3.15 ; 95 \%$ CI 1.87 to $5.33, p<0.001$ ) were significantly associated with an increased risk. Advanced age (OR 0.95; 95\% CI 0.93 to $0.97 ; p<0.001)$ was protective for symptomatic gallstone disease after bariatric surgery, and UDCA prophylaxis did not reach statistical significance (OR 0.64; 95\% CI 0.39 to $1.03 ; p=0.07$ ). Similar associations were observed in patients assigned to placebo (Supplementary Table 1). A significant interaction between the intervention (UDCA/placebo) and the presence of asymptomatic gallstones before surgery was identified in the univariable analysis (OR 2.83; 95\% CI 1.02 to $7.86 ; p=0.046)$. The final model of the multivariable analysis including this interaction is shown in Supplementary Table 2. Age and the presence of a pain

Fig. 1 Flow diagram

967 patients were included in the study population of the UPGRADE trial

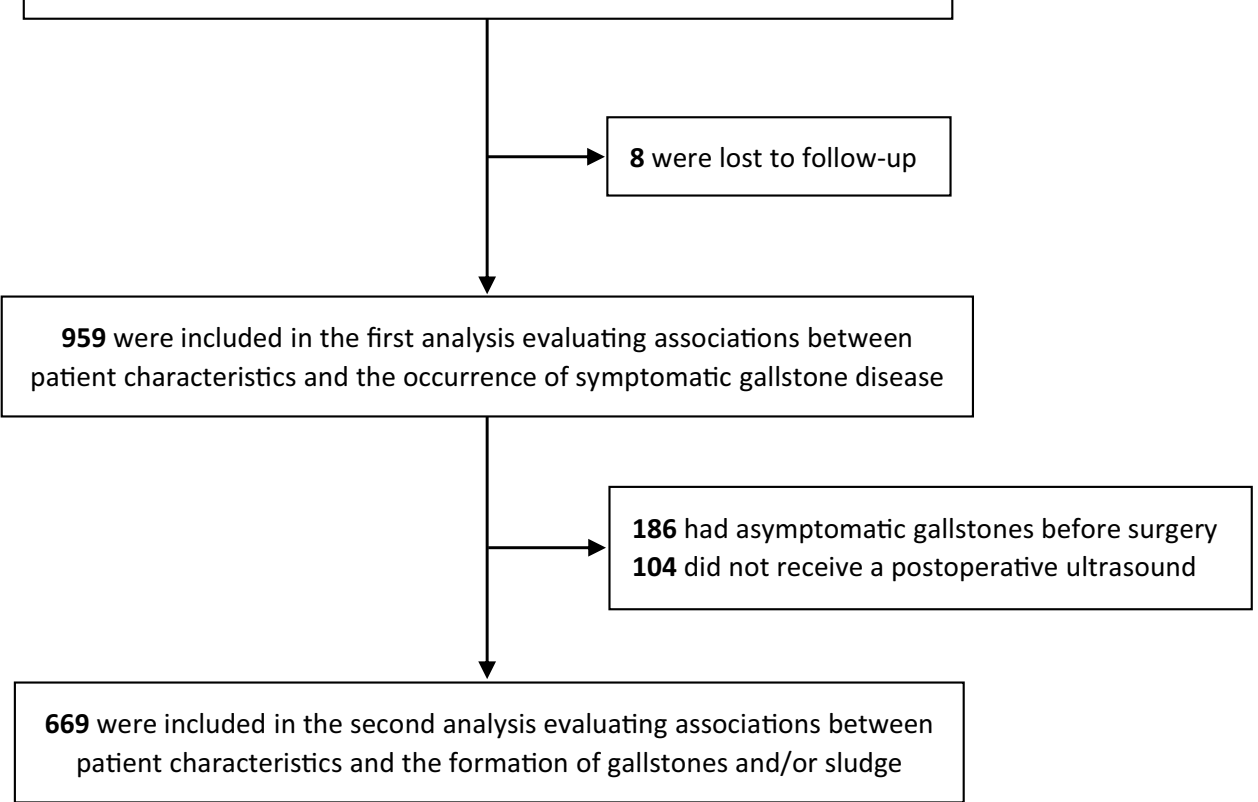


Table 1 Demographic and clinical characteristics of the total study population and a subset consisting of patients without gallstones before surgery who received a postoperative ultrasound

\begin{tabular}{|c|c|c|}
\hline & $\begin{array}{l}\text { Study population } \\
(n=959)\end{array}$ & Subset $(n=669)$ \\
\hline \multicolumn{3}{|l|}{ Preoperative } \\
\hline Age - years & $45.1 \pm 11.1$ & $44.8 \pm 11.3$ \\
\hline Female gender $-n(\%)$ & $766(80)$ & $519(78)$ \\
\hline \multicolumn{3}{|l|}{ Ethnicity $-n(\%)$} \\
\hline - Dutch & $734(77)$ & $499(75)$ \\
\hline - Surinamese & $55(6)$ & $45(7)$ \\
\hline - Dutch Caribbean & $28(3)$ & $21(3)$ \\
\hline - Moroccan & $18(2)$ & $14(2)$ \\
\hline - Turkish & $16(2)$ & $11(2)$ \\
\hline - Other & $49(5)$ & $40(6)$ \\
\hline - Multiple & $59(6)$ & $39(6)$ \\
\hline Weight at surgery - kg & $116.0 \pm 18.0$ & $115.7 \pm 18.3$ \\
\hline Body mass index at surgery $-\mathrm{kg} / \mathrm{m}^{2}$ & $40.2 \pm 4.7$ & $40.1 \pm 4.8$ \\
\hline Hypertension $-n(\%)$ & $468(49)$ & $311(46)$ \\
\hline Dyslipidemia $-n(\%)$ & $301(31)$ & $208(31)$ \\
\hline Type 2 diabetes $-n(\%)$ & $156(16)$ & $111(17)$ \\
\hline Pain syndrome $-n(\%)$ & $81(8)$ & $61(9)$ \\
\hline Asymptomatic gallstones before surgery $-n(\%)$ & $186(19)$ & NA \\
\hline \multicolumn{3}{|l|}{ Type of surgery $-n(\%)$} \\
\hline - RYGB & $881(92)$ & $611(91)$ \\
\hline - Sleeve gastrectomy & $78(8)$ & $58(9)$ \\
\hline Statin use $-n(\%)$ & $164(17)$ & $122(18)$ \\
\hline - Low intensity & $26(3)$ & $21(3)$ \\
\hline - Moderate intensity & $85(9)$ & $64(10)$ \\
\hline - High intensity & $53(6)$ & $37(6)$ \\
\hline \multicolumn{3}{|l|}{ Postoperative } \\
\hline Assigned to UDCA $-n(\%)$ & $475(50)$ & $331(50)$ \\
\hline$\% \mathrm{TWL}$ at 6 months* - percent & $23.0 \pm 5.4$ & $23.0 \pm 5.4$ \\
\hline$\% \mathrm{TWL}$ at 12 months* - percent & $29.4 \pm 7.4$ & $29.4 \pm 7.5$ \\
\hline$\%$ TWL at 24 months* - percent & $29.4 \pm 8.9$ & $29.4 \pm 9.2$ \\
\hline
\end{tabular}

Plus-minus values are means \pm standard deviations. $N$, number; $R Y G B$, Roux-en-Y gastric bypass; $U D C A$, ursodeoxycholic acid; $T W L$, total weight loss. ${ }^{*}$ In the study population, data on weight was missing in 9 patients at 6 months, in 8 patients at 12 months, and in 5 patients at 24 months. In the subset, data on weight was missing in 1 patient at 6 and 12 months syndrome were similarly associated with the risk for symptomatic gallstone disease after bariatric surgery as compared to the multivariable model without the interaction term. The effect of UDCA prophylaxis was significant in patients without gallstones before surgery (OR 0.46 ; 95\% CI 0.25-0.85; $p=0.01)$.

\section{Factors Associated with the Formation of Gallstones and/or Sludge}

Gallstones and/or sludge were observed on the follow-up ultrasound in 107 (16\%) of the 669 patients without gallstones before surgery, of whom 56 (52\%) were asymptomatic. The results of the univariable and multivariable logistic regression analyses for the outcome formation of gallstones and/or sludge are shown in Table 3. None of the patient characteristics was significantly associated with an increased risk for the formation of gallstones and/or sludge, although type 2 diabetes almost reached significance in the multivariable analysis (OR $1.80 ; 95 \%$ CI 0.97 to 3.34 ; $p=0.06)$. In both the univariable and multivariable analyses, advanced age (OR $0.98 ; 95 \%$ CI 0.96 to $1.00 ; p=0.04$ ), the use of statins (OR $0.42 ; 95 \%$ CI 0.20 to $0.90 ; p=0.03$ ), and UDCA prophylaxis (OR 0.47 ; $95 \%$ CI 0.30 to 0.73 ; $p<0.001)$ were significantly associated with a reduced risk. No dose-effect relationship was observed between the intensity of the statins and the formation of gallstones and/ or sludge $(p=0.13)$. In patients assigned to placebo, only the use of statins was significantly associated with the formation of gallstones and/or sludge in the multivariable analysis 
Table 2 Association between patient characteristics and symptomatic gallstone disease in the study population $(n=959)$

\begin{tabular}{|c|c|c|c|c|}
\hline & \multicolumn{2}{|l|}{ Univariable } & \multicolumn{2}{|l|}{ Multivariable $\dagger$} \\
\hline & OR $(95 \% \mathrm{CI})$ & $p$-value & OR $(95 \% \mathrm{CI})$ & $p$-value \\
\hline \multicolumn{5}{|l|}{ Preoperative } \\
\hline Age - years & $0.96(0.94$ to 0.98$)$ & $<0.001$ & $0.95(0.93$ to 0.97$)$ & $<0.001$ \\
\hline Gender - female vs male & $1.78(0.90$ to 3.53$)$ & 0.10 & - & \\
\hline Weight at surgery $-\mathrm{kg}$ & $1.00(0.98$ to 1.01$)$ & 0.59 & $\mathrm{x}^{*}$ & \\
\hline Body mass index at surgery $-\mathrm{kg} / \mathrm{m}^{2}$ & $1.00(0.95$ to 1.05$)$ & 0.92 & $\mathrm{x}^{*}$ & \\
\hline Hypertension - yes vs. no & $0.63(0.39$ to 1.02$)$ & 0.06 & - & \\
\hline Dyslipidemia - yes vs. no & $0.59(0.34$ to 1.02$)$ & 0.06 & - & \\
\hline Type 2 diabetes - yes vs. no & $0.48(0.22$ to 1.07$)$ & 0.07 & - & \\
\hline Pain syndrome - yes vs. no & $1.90(0.96$ to 3.76$)$ & 0.06 & 2.07 (1.03 to 4.17$)$ & 0.04 \\
\hline Asymptomatic gallstones before surgery - yes vs. no & $2.40(1.46$ to 3.95$)$ & $<0.001$ & $3.15(1.87$ to 5.33$)$ & $<0.001$ \\
\hline Type of surgery - RYGB vs. sleeve gastrectomy & $0.65(0.31$ to 1.36$)$ & 0.25 & - & \\
\hline Statin use - yes vs. no & $0.38(0.16$ to 0.89$)$ & 0.03 & - & \\
\hline \multicolumn{5}{|l|}{ Postoperative } \\
\hline Intervention - UDCA vs. placebo & $0.65(0.40$ to 1.04$)$ & 0.07 & $0.64(0.39$ to 1.03$)$ & 0.07 \\
\hline$\%$ TWL at 6 months - percent & $1.04(0.99$ to 1.08$)$ & 0.10 & - & \\
\hline$\% \mathrm{TWL}$ at 12 months - percent & $1.03(1.00$ to 1.06$)$ & 0.09 & $\mathrm{x}^{*}$ & \\
\hline$\% \mathrm{TWL}$ at 24 months - percent & $1.03(1.00$ to 1.06$)$ & 0.04 & $\mathrm{x}^{*}$ & \\
\hline
\end{tabular}

$O R$, odds ratio; $C I$, confidence interval; $R Y G B$, Roux-en-Y gastric bypass; $U D C A$, ursodeoxycholic acid; $T W L$, total weight loss. *Variables not included in the multivariable model. $\uparrow$ Variables excluded from the model following stepwise backward elimination: gender, hypertension, dyslipidemia, type 2 diabetes, type of surgery, statin use, and \%TWL at 6 months

Table 3 Association between patient characteristics and the formation of gallstones and/or sludge in the subset $(n=669)$

\begin{tabular}{|c|c|c|c|c|}
\hline & \multicolumn{2}{|l|}{ Univariable } & \multicolumn{2}{|l|}{ Multivariable $\dagger$} \\
\hline & OR $(95 \% \mathrm{CI})$ & $p$-value & OR $(95 \% \mathrm{CI})$ & $p$-value \\
\hline \multicolumn{5}{|l|}{ Preoperative } \\
\hline Age - years & 0.97 (0.96 to 0.99$)$ & 0.006 & $0.98(0.96$ to 1.00$)$ & 0.04 \\
\hline Gender - female vs male & $1.14(0.69$ to 1.89$)$ & 0.61 & - & \\
\hline Weight at surgery $-\mathrm{kg}$ & $1.00(0.99$ to 1.01$)$ & 0.60 & $\mathrm{x}^{*}$ & \\
\hline Body mass index at surgery $-\mathrm{kg} / \mathrm{m}^{2}$ & $1.02(0.97$ to 1.06$)$ & 0.49 & $\mathrm{x}^{*}$ & \\
\hline Hypertension - yes vs. no & $0.81(0.53$ to 1.23$)$ & 0.32 & - & \\
\hline Dyslipidemia - yes vs. no & $0.67(0.42$ to 1.08$)$ & 0.10 & - & \\
\hline Type 2 diabetes - yes vs. no & $1.19(0.70$ to 2.03$)$ & 0.52 & $1.80(0.97$ to 3.34$)$ & 0.06 \\
\hline Pain syndrome - yes vs. no & $1.48(0.77$ to 2.84$)$ & 0.24 & - & \\
\hline Type of surgery - RYGB vs. sleeve gastrectomy & $0.80(0.40$ to 1.59$)$ & 0.52 & - & \\
\hline Statin use - yes, no (\%) & $0.47(0.24$ to 0.90$)$ & 0.02 & $0.42(0.20$ to 0.90$)$ & 0.03 \\
\hline \multicolumn{5}{|l|}{ Postoperative } \\
\hline Intervention - UDCA vs. placebo & $0.46(0.30$ to 0.71$)$ & $<0.001$ & $0.47(0.30$ to 0.73$)$ & $<0.001$ \\
\hline$\% \mathrm{TWL}$ at 6 months - percent & $1.00(0.97$ to 1.04$)$ & 0.85 & - & \\
\hline$\%$ TWL at 12 months - percent & $1.00(0.97$ to 1.03$)$ & 0.90 & $\mathrm{x}^{*}$ & \\
\hline$\% \mathrm{TWL}$ at 24 months - percent & $1.01(0.99$ to 1.03$)$ & 0.42 & $x^{*}$ & \\
\hline
\end{tabular}

$O R$, odds ratio; $C I$, confidence interval; $R Y G B$, Roux-en-Y gastric bypass; UDCA, ursodeoxycholic acid; $T W L$, total weight loss. *Variables not included in the multivariable model. †Variables excluded from the model following stepwise backward elimination: gender, hypertension, dyslipidemia, pain syndrome, type of surgery, and \%TWL at 6 months 
(Supplementary Table 3). A significant interaction between the intervention and the presence of type 2 diabetes was identified in the univariable analysis (OR 3.79; 95\% CI 1.27 to $11.31 ; p=0.02)$. The final model of the multivariable analysis including this interaction is shown in Supplementary Table 4. Age and statin use were similarly associated with the formation of gallstones and/or sludge as compared to the multivariable model without the interaction term. The protective effect of UDCA prophylaxis was stronger in patients without type 2 diabetes compared to the entire subset (OR 0.37; 95\% CI 0.23-0.61; $p<0.001$ ).

\section{Discussion}

In this prospective cohort study, we investigated associations between patient characteristics and the occurrence of symptomatic gallstone disease, and the formation of gallstones and/or sludge in patients who underwent bariatric surgery. Risk factors for symptomatic gallstone disease after bariatric surgery were a pain syndrome and the presence of asymptomatic gallstones before surgery, whereas advanced age was a protective factor. Besides UDCA prophylaxis, advanced age and the use of statins were found to reduce the formation of gallstones and/or sludge.

Obesity is a well-established risk factor for cholesterol gallstone disease. Consequently, asymptomatic gallstones are frequently encountered in patients scheduled for bariatric surgery. In this study, 186 (19\%) patients had asymptomatic gallstones before surgery. Patients and treating physicians were both blinded for the preoperative ultrasound result, and therefore not aware of the presence of gallstones. Still, patients with asymptomatic gallstones before surgery were more likely to develop symptomatic gallstone disease afterwards than those without. Three retrospective cohort studies also included patients with asymptomatic gallstones and investigated their association with biliary complications or cholecystectomy after bariatric surgery [18, 26, 27]. In contrast to our finding, two of them did not observe a significant association [18, 27], while Chang et al. only identified a significant association in the univariable analysis [26]. The variation in study design, study population, and/or method for statistical analysis might explain the difference. Moreover, theoretically it also makes sense that patients with asymptomatic gallstones before surgery have an increased risk of developing symptomatic gallstones disease after bariatric surgery. First, they are at risk for the progression of asymptomatic to symptomatic disease, regardless of the bariatric procedure. Second, the bariatric procedure might foster the formation of new gallstones and/or sludge in already susceptible patients. Third, since abdominal complaints are common postoperatively and abdominal ultrasounds are frequently performed, there is a risk that misclassification may also contribute. Gallstones are by definition present in patients who had asymptomatic gallstones before surgery and therefore can be regarded as potential cause of symptoms. Among patients without gallstones before surgery, this can only be the case in those who develop new stones. Howbeit, no preventive measures are currently available for patients with asymptomatic gallstones before surgery. Prophylactic cholecystectomy is not warranted, because the majority of patients remains asymptomatic afterwards [7]. Likewise, UDCA prophylaxis does not seem to be indicated, since we have shown that it did not reduce the occurrence of symptomatic gallstone disease in these patients [41].

The presence of a pain syndrome was also identified as risk factor for the occurrence of symptomatic gallstone disease after bariatric surgery, which is in line with our previous finding that a pain syndrome increases the chance of undergoing cholecystectomy after bariatric surgery [30] In our previous paper, we speculated that patients with a pain syndrome are more likely to visit a doctor in case they experience abdominal pain, often resulting in imaging of the abdomen. We all know that postoperatively, the probability to find gallstones during imaging is high. Hence, a very thorough diagnostic workup of abdominal complaints after bariatric surgery should be done in order to accurately diagnose symptomatic gallstones disease to prevent unnecessary cholecystectomies. Accordingly, we found no association between the presence of a pain syndrome and the formation of gallstones and/or sludge, since patients received the postoperative ultrasound for study purposes rather than complaints.

In the present study, we did not find a significant independent association between \% TWL and symptomatic gallstone disease, nor between \% TWL and the formation of gallstones and/or sludge. At first, this may seem contradictory since rapid weight loss is a well-established risk factor for cholesterol gallstone disease, and the reason why bariatric patients are at risk. However, the fact that all bariatric patients lose weight might explain why we did not find an association in our cohort. A thorough literature search yielded inconsistent findings. Like our study, many studies did not find a significant association between weight loss and gallstone disease in a bariatric population [2-4, 10, 12, 14, 16-19, 22-26, 28, 29, 32, 33, 37-39], whereas several others did $[8,9,11,13,15,21,27,30,35,36,44]$. Hence, it seems unlikely that \%TWL (or another weight loss outcome measure) can be of help to identify specific bariatric patients at risk and to guide preventive treatment in the near future.

In the general population, metabolic abnormalities such as dyslipidemia, type 2 diabetes, and insulin resistance predispose patients to cholesterol gallstone disease [1]. In our study, dyslipidemia and type 2 diabetes were not significantly associated with our study outcomes. Hypothetically, it is unlikely that the presence of preoperative comorbidities 
could play a major role in the prediction of which bariatric patient is at high risk, since their existence is rapidly decreased after the bariatric procedure.

In line with several other studies who have shown that advanced age decreases the risk on cholecystectomy after bariatric surgery [30, 31, 36, 37], we have found that older patients have a decreased risk on symptomatic gallstone disease and gallstone/sludge formation.

Apart from UDCA prophylaxis as being protective in patients without gallstones before surgery, lipid-lowering drugs might also be able to reduce gallstone disease and gallstone formation after bariatric surgery. Our univariable analyses showed that preoperative statin use significantly reduced the occurrence of symptomatic gallstone disease and the formation of gallstones and/or sludge after bariatric surgery. However, using stepwise backward elimination, statin use was only maintained in the multivariable model for the formation of gallstones and/or sludge. In contrast to our previous retrospective study, we did not observe a dose-effect relationship [30]. Yet, these results should be confirmed in a prospective intervention study.

Some studies have reported that in the general population, the prevalence of gallstone disease varies by ethnic group as the result of different genetic susceptibility and environmental factors [1]. Therefore, ethnicity might be associated with our outcomes. However, the non-Dutch ethnic groups were too small to properly evaluate this association. Moreover, an etiological principle why ethnicity would differentially affect the risk of gallstones in an obese population during rapid weight loss seems lacking.

Strengths of this cohort study include the prospective data collection, blinded endpoint adjudication, and the low number of losses to follow-up in the UPGRADE trial, which resulted in a valid and complete data set. On the other hand, the use of randomized trial data might have introduced some selection bias because of the entry criteria, and the willingness to participate [45]. However, we have no indications that we have selected healthier participants, since the exclusion criteria were not extensive.

\section{Conclusion}

Our findings suggest that young patients with a preoperative pain syndrome and/or asymptomatic gallstones before surgery are at increased risk for symptomatic gallstone disease after bariatric surgery. A prospective study to explore whether statins or other lipid-lowering drugs, either alone or in combination with UDCA prophylaxis, further reduce the burden of gallstones after bariatric surgery is desirable. Following this study, the development of an useful clinical prediction model will be a step forward in a tailored approach for the prevention of symptomatic gallstone disease and gallstone formation after bariatric surgery.

Supplementary Information The online version contains supplementary material available at https://doi.org/10.1007/s11695-022-05947-8.

Funding This study was funded by the Netherlands Organization for Health Research and Development (grant number 848015003), Zambon Netherlands BV, Foundation for Clinical Research of the Slotervaart Hospital, Spaarne Gasthuis Academy, and Amsterdam Gastroenterology Endocrinology Metabolism.

\section{Declarations}

Ethical Approval All procedures performed in studies involving human participants were in accordance with the ethical standards of the institutional and/or national research committee and with the 1964 Helsinki declaration and its later amendments or comparable ethical standards.

Informed Consent Informed consent was obtained from all individual participants included in the study.

Conflict of Interest The authors declare no competing interests.

Open Access This article is licensed under a Creative Commons Attribution 4.0 International License, which permits use, sharing, adaptation, distribution and reproduction in any medium or format, as long as you give appropriate credit to the original author(s) and the source, provide a link to the Creative Commons licence, and indicate if changes were made. The images or other third party material in this article are included in the article's Creative Commons licence, unless indicated otherwise in a credit line to the material. If material is not included in the article's Creative Commons licence and your intended use is not permitted by statutory regulation or exceeds the permitted use, you will need to obtain permission directly from the copyright holder. To view a copy of this licence, visit http://creativecommons.org/licenses/by/4.0/.

\section{References}

1. Portincasa P, Moschetta A, Palasciano G. Cholesterol gallstone disease. Lancet. 2006;368(9531):230-9.

2. Adams LB, Chang C, Pope J, et al. Randomized, prospective comparison of ursodeoxycholic acid for the prevention of gallstones after sleeve gastrectomy. Obes Surg. 2016;26(5):990-4.

3. Nabil TM, Khalil AH, Gamal K. Effect of oral ursodeoxycholic acid on cholelithiasis following laparoscopic sleeve gastrectomy for morbid obesity. Surg Obes Relat Dis. 2019;15(6):827-31.

4. Sakran N, Dar R, Assalia A, et al. The use of Ursolit for gallstone prophylaxis following bariatric surgery: a randomized-controlled trial. Updates Surg. 2020;72(4):1125-33.

5. Altieri MS, Yang J, Nie L, et al. Incidence of cholecystectomy after bariatric surgery. Surg Obes Relat Dis. 2018;14(7):992-6.

6. Coupaye M, Calabrese D, Sami O, et al. Evaluation of incidence of cholelithiasis after bariatric surgery in subjects treated or not treated with ursodeoxycholic acid. Surg Obes Relat Dis. 2017;13(4):681-5.

7. Portenier DD, Grant JP, Blackwood HS, et al. Expectant management of the asymptomatic gallbladder at Roux-en-Y gastric bypass. Surg Obes Relat Dis. 2007;3(4):476-9. 
8. Aldriweesh MA, Aljahdali GL, Shafaay EA, et al. The incidence and risk factors of cholelithiasis development after bariatric surgery in saudi arabia: a two-center retrospective cohort study. Front Surg. 2020;7:559064.

9. Alsaif FA, Alabdullatif FS, Aldegaither MK, et al. Incidence of symptomatic cholelithiasis after laparoscopic sleeve gastrectomy and its association with rapid weight loss. Saudi J Gastroenterol. 2020;26(2):94-8.

10. Coupaye M, Calabrese D, Sami O, et al. Effectiveness of ursodeoxycholic acid in the prevention of cholelithiasis after sleeve gastrectomy. Obes Surg. 2019;29(8):2464-9.

11. Coupaye M, Castel B, Sami O, et al. Comparison of the incidence of cholelithiasis after sleeve gastrectomy and Roux-en-Y gastric bypass in obese patients: a prospective study. Surg Obes Relat Dis. 2015;11(4):779-84.

12. de IgleziasBrandao Oliveira C, Adami Chaim E, da Silva BB. Impact of rapid weight reduction on risk of cholelithiasis after bariatric surgery. Obes Surg. 2003;13(4):625-8.

13. Li VK, Pulido N, Fajnwaks P, et al. Predictors of gallstone formation after bariatric surgery: a multivariate analysis of risk factors comparing gastric bypass, gastric banding, and sleeve gastrectomy. Surg Endosc. 2009;23(7):1640-4.

14. Machado FHF, Castro Filho HF, Babadopulos R, et al. Ursodeoxycholic acid in the prevention of gallstones in patients subjected to Roux-en-Y gastric bypass1. Acta Cir Bras. 2019;34(1):e20190010000009.

15. Melmer A, Sturm W, Kuhnert B, et al. Incidence of gallstone formation and cholecystectomy 10 years After Bariatric Surgery. Obes Surg. 2015;25(7):1171-6.

16. Nagem R, Lazaro-da-Silva A. Cholecystolithiasis after gastric bypass: a clinical, biochemical, and ultrasonographic 3-year follow-up study. Obes Surg. 2012;22(10):1594-9.

17. Pizza F, D'Antonio D, Lucido FS, et al. The role of ursodeoxycholic acid (UDCA) in cholelithiasis management after one anastomosis gastric bypass (OAGB) for morbid obesity: results of a monocentric randomized controlled trial. Obes Surg. 2020;30(11):4315-24.

18. Rottenstreich A, Kleinstern G, Levin G, et al. Does pregnancy increase the risk of cholecystectomy following bariatric surgery? A cross-sectional cohort study. Surg Obes Relat Dis. 2019;15(10):1822-8.

19. Talha A, Abdelbaki T, Farouk A, et al. Cholelithiasis after bariatric surgery, incidence, and prophylaxis: randomized controlled trial. Surg Endosc. 2020;34(12):5331-7.

20. Villegas L, Schneider B, Provost D, et al. Is routine cholecystectomy required during laparoscopic gastric bypass? Obes Surg. 2004;14(2):206-11.

21. Vural A, Goksu K, Kahraman AN, et al. Increased gallstone formation after sleeve gastrectomy and the preventive role of ursodeoxycholic acid. Acta Gastroenterol Belg. 2020;83(1):33-8.

22. Worobetz LJ, Inglis FG, Shaffer EA. The effect of ursodeoxycholic acid therapy on gallstone formation in the morbidly obese during rapid weight loss. Am J Gastroenterol. 1993;88(10):1705-10.

23. Abdallah E, Emile SH, Elfeki H, et al. Role of ursodeoxycholic acid in the prevention of gallstone formation after laparoscopic sleeve gastrectomy. Surg Today. 2017;47(7):844-50.

24. Alimoğulları M, Buluş H. Predictive factors of gallstone formation after sleeve gastrectomy: a multivariate analysis of risk factors. Surg Today. 2020;50(9):1002-1007.

25. Cazzo E, Gestic MA, Utrini MP, et al. Influence of insulin resistance status on the development of gallstones following rouxen-y gastric bypass: a prospective cohort study. Obes Surg. 2016;26(4):769-75.
26. Chang J, Corcelles R, Boules M, et al. Predictive factors of biliary complications after bariatric surgery. Surg Obes Relat Dis. 2016;12(9):1706-10.

27. D'Hondt M, Sergeant G, Deylgat B, et al. Prophylactic cholecystectomy, a mandatory step in morbidly obese patients undergoing laparoscopic Roux-en-Y gastric bypass? J Gastrointest Surg. 2011;15(9):1532-6.

28. Dakour Aridi H, Sultanem S, Abtar H, et al. Management of gallbladder disease after sleeve gastrectomy in a selected Lebanese population. Surg Obes Relat Dis. 2016;12(7):1300-4.

29. Guzmán HM, Sepúlveda M, Rosso N, et al. Incidence and risk factors for cholelithiasis after bariatric surgery. Obes Surg. 2019;29(7):2110-4.

30. Haal S, Rondagh D, Hutten BA, et al. Risk factors for cholecystectomy after laparoscopic Roux-En-Y gastric bypass. Obes Surg. 2020;30(2):507-14.

31. Juo YY, Khrucharoen U, Chen Y, et al. Cost analysis and risk factors for interval cholecystectomy after bariatric surgery: a national study. Surg Obes Relat Dis. 2018;14(3):368-74.

32. Manatsathit $\mathrm{W}$, Leelasincharoen $\mathrm{P}, \mathrm{Al}-\mathrm{Hamid} \mathrm{H}$, et al. The incidence of cholelithiasis after sleeve gastrectomy and its association with weight loss: a two-centre retrospective cohort study. Int J Surg. 2016;30:13-8.

33. Özdaş S, Bozkurt H. Factors affecting the development of gallstones following laparoscopic sleeve gastrectomy. Obes Surg. 2019;29(10):3174-8.

34. Sneineh MA, Harel L, Elnasasra A, et al. Increased Incidence of symptomatic cholelithiasis after bariatric Roux-En-Y Gastric bypass and previous bariatric surgery: a single center experience. Obes Surg. 2020;30(3):846-50.

35. Tarantino I, Warschkow R, Steffen T, et al. Is routine cholecystectomy justified in severely obese patients undergoing a laparoscopic Roux-en-Y gastric bypass procedure? A comparative cohort study. Obes Surg. 2011;21(12):1870-8.

36. Tsirline VB, Keilani ZM, El Djouzi S, et al. How frequently and when do patients undergo cholecystectomy after bariatric surgery? Surg Obes Relat Dis. 2014;10(2):313-21.

37. Wanjura V, Sandblom G, Österberg J, et al. Cholecystectomy after gastric bypass-incidence and complications. Surg Obes Relat Dis. 2017;13(6):979-87.

38. Morais M, Faria G, Preto J, Costa-Maia J. Gallstones and bariatric surgery: to treat or not to treat? World J Surg. 2016;40(12):2904-10.

39. Shiffman ML, Sugerman HJ, Kellum JM, et al. Gallstone formation after rapid weight loss: a prospective study in patients undergoing gastric bypass surgery for treatment of morbid obesity. Am J Gastroenterol. 1991;86(8):1000-5.

40. Boerlage TCC, Haal S, de Brauw LM, et al. Ursodeoxycholic acid for the prevention of symptomatic gallstone disease after bariatric surgery: study protocol for a randomized controlled trial (UPGRADE trial). BMC Gastroenterol. 2017;17(1):164.

41. Haal S, Guman MSS, Boerlage TCC, et al. Ursodeoxycholic acid for the prevention of symptomatic gallstone disease after bariatric surgery (UPGRADE): a multicentre, double-blind, randomised, placebo-controlled superiority trial. Lancet Gastroenterol Hepatol. 2021;6(12):993-1001.

42. Castor EDC. Castor electronic data capture 2019 [27 Aug. 2019]. Available from: https://castoredc.com.

43. Law MR, Wald NJ, Rudnicka AR. Quantifying effect of statins on low density lipoprotein cholesterol, ischaemic heart disease, and stroke: systematic review and meta-analysis. BMJ. 2003;326(7404):1423. 
44. Wudel LJ Jr, Wright JK, Debelak JP, et al. Prevention of gallstone formation in morbidly obese patients undergoing rapid weight loss: results of a randomized controlled pilot study. J Surg Res. 2002;102(1):50-6.

45. Collins GS, Reitsma JB, Altman DG, et al. Transparent reporting of a multivariable prediction model for individual prognosis or diagnosis (TRIPOD): the TRIPOD statement. Bmj. 2015;350:g7594.

Publisher's Note Springer Nature remains neutral with regard to jurisdictional claims in published maps and institutional affiliations.

\section{Authors and Affiliations}

\section{Sylke Haal ${ }^{1,2} \mathbb{1} \cdot$ Maimoena S. S. Guman ${ }^{1,3} \cdot$ Sjoerd Bruin $^{4} \cdot$ Ruben Schouten $^{5} \cdot$ Ruben N. van Veen $^{6} \cdot$ Paul Fockens $^{2}$. Marcel G. W. Dijkgraaf ${ }^{7} \cdot$ Barbara A. Hutten $^{8} \cdot$ Victor E. A. Gerdes $^{1,3} \cdot$ Rogier P. Voermans $^{2}$}

1 Department of Internal Medicine, Spaarne Gasthuis, 2134 TM Hoofddorp, the Netherlands

2 Department of Gastroenterology and Hepatology, Amsterdam Gastroenterology Endocrinology Metabolism, Amsterdam UMC, University of Amsterdam, 1105 AZ Amsterdam, the Netherlands

3 Department of Internal and Vascular Medicine, Amsterdam Gastroenterology Endocrinology Metabolism, Amsterdam UMC, University of Amsterdam, 1105 AZ Amsterdam, the Netherlands

4 Department of Surgery, Spaarne Gasthuis, 2134 TM Hoofddorp, the Netherlands
5 Department of Surgery, Flevohospital, 1315 RA Almere, the Netherlands

6 Department of Surgery, OLVG, 1061 AE Amsterdam, the Netherlands

7 Department of Epidemiology and Data Science, Amsterdam UMC, University of Amsterdam, 1105 AZ Amsterdam, the Netherlands

8 Department of Epidemiology and Data Science, Amsterdam UMC, University of Amsterdam, Amsterdam Cardiovascular Sciences, 1105 AZ Amsterdam, the Netherlands 